\title{
Stem Cell-Based Therapy in Neural Repair
}

\author{
Yi-Chao Hsu ${ }^{1}$, Su-Liang Chen ${ }^{1}$, Dan-Yen Wang ${ }^{1,2}$, Ing-Ming Chiu ${ }^{1,2}$
}

\begin{abstract}
Cell-based therapy could aid in alleviating symptoms or even reversing the progression of neurodegenerative diseases and nerve injuries. Fibroblast growth factor 1 (FGF1) has been shown to maintain the survival of neurons and induce neurite outgrowth. Accumulating evidence suggests that combination of FGF1 and cell-based therapy is promising for future therapeutic application. Neural stem cells (NSCs), with the characteristics of self-renewal and multipotency, can be isolated from embryonic stem cells, embryonic ectoderm, and developing or adult brain tissues. For NSC clinical application, several critical problems remain to be resolved: (1) the source of NSCs should be personalized; (2) the isolation methods and protocols of human NSCs should be standardized; (3) the clinical efficacy of NSC transplants must be evaluated in more adequate animal models; and (4) the mechanism of intrinsic brain repair needs to be better characterized. In addition, the ideal imaging technique for tracking

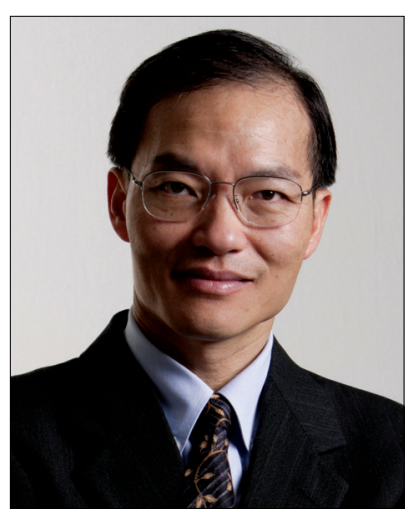

Prof. Ing-Ming Chiu NSCs would be safe and yield high temporal and spatial resolution, good sensitivity and specificity. Here, we discuss recent progress and future development of cell-based therapy, such as NSCs, induced pluripotent stem cells, and induced neurons, in neurodegenerative diseases and peripheral nerve injuries. (Biomed J 2013;36:98-105)
\end{abstract}

\section{Key words: cell therapy, fibroblast growth factor 1, induced neurons, neural stem cells, neuroregeneration, peripheral nerve injury}

\section{Neurodegenerative diseases}

$\mathrm{T}_{\mathrm{s}}^{\mathrm{h}}$ he prevalence of neurodegenerative diseases is increasing rapidly. Neurodegenerative diseases such as Parkinson's disease (PD), Huntington's disease (HD), and Alzheimer's disease (AD) trigger neuronal cell death through endogenous suicide pathways. Although progressive neuronal loss is a hallmark of neurodegenerative disorders, some neurological impairment may reflect dysfunction rather than loss of neurons. Abnormal protein assemblies seem to trigger vicious cycles of aberrant neuronal activity and compensatory alterations in neurotransmitter receptors and related signaling pathways that lead to synaptic deficits, disintegration of neural networks, and ultimately, failure of neurological functions.

Recently, the US President Obama's administration plans to spend an additional $\$ 156$ million over the next 2 years to help find an effective treatment for AD that affects more than 5 million Americans. The spending increase is intended help make good on a US target set last month to find a way to treat or prevent AD by 2025 . Current drugs help manage symptoms, but so far no therapy can stop the progression of $\mathrm{AD}$, which can start with vague memory loss and confusion before progressing to complete disability and death. Experts predict that without an effective treatment, the number of Americans with AD will double by 2050 and related healthcare costs could soar to more than $\$ 1$ trillion a year.

Neurodegenerative diseases can disrupt molecular pathways, synapses, neuronal subpopulations, and local circuits in specific brain regions, as well as higher-order neural networks. Abnormal network activities may result in a vicious cycle, further impairing the integrity and functions of neurons and synapses, for example, through aberrant excitation or inhibition. Neurodegenerative disorders such as $\mathrm{AD}$ and amyotrophic lateral sclerosis (ALS) are associated with microvascular dysfunction and/or degeneration in the

From the ${ }^{1}$ Division of Regenerative Medicine, Institute of Cellular and System Medicine, National Health Research Institutes, Miaoli, Taiwan; ${ }^{2}$ Institute of Molecular Medicine and Department of Life Science, National Tsing Hua University, Hsinchu, Taiwan Received: Jul. 10, 2012; Accepted: Aug. 16, 2012

Correspondence to: Prof. Ing-Ming Chiu, Division of Regenerative Medicine, Institute of Cellular and System Medicine, National Health Research Institutes. 35, Keyan Road, Zhunan, Miaoli 350, Taiwan (R.O.C.). Tel: 886-37-246166 ext. 37501, Fax: 886-37-587408; E-mail: ingming@ nhri.org.tw

DOI: $10.4103 / 2319-4170.113226$ 
brain, neurovascular disintegration, defective blood-brain barrier function and/or vascular factors. Microvascular deficits diminish cerebral blood flow and, consequently, the brain's supply of oxygen, energy substrates, and nutrients. Moreover, such deficits impair the clearance of neurotoxic molecules, non-neuronal cells, and neurons. Recent evidence suggests that vascular dysfunction leads to neuronal dysfunction and neurodegeneration, and that it might contribute to the development of proteinaceous brain and cerebrovascular "storage" disorders. Such disorders include cerebral $\beta$-amyloidosis and cerebral amyloid angiopathy, which are caused by accumulation of the peptide $\beta$-amyloid in the brain and the vessel wall, respectively, and are features of AD. ${ }^{[1]}$

$\mathrm{PD}$ is a degenerative disorder of the central nervous system. It was first described in 1817 by James Parkinson. Researchers believe that at least 500,000 people in the United States currently have PD, although some estimates are even higher. Society pays an enormous price for PD. The total cost to the nation is estimated to exceed $\$ 6$ billion annually. The risk of PD increases with age, so analysts expect the financial and public health impact of this disease to increase as the population gets older. PD is characterized by an extensive loss of dopaminergic neurons in the substantia nigra, pars compacta, and their terminals in the striatum. ${ }^{[2]}$ Although the etiology of idiopathic PD is not known, several predisposing factors for the dopaminergic depletion associated with the disease have been suggested, including programmed cell death, viral infection, and environmental toxins. As an effective treatment for PD, patients have been given L-dihydroxyphenylalanine (L-DOPA), a precursor of dopamine, but long-term administration of L-DOPA consequently produces grave side effects.

\section{Peripheral nerve injuries}

The incidence of peripheral nerve injury (PNI) in developed countries is estimated between 13 and 23 per 100,000 persons per year. Injuries to peripheral nerves result in partial or total loss of motor, sensory, and autonomic functions in the involved segments of the body. Reinnervation of denervated targets can be achieved by regeneration of injured axons or by collateral branching of undamaged axons in the vicinity. Nevertheless, these mechanisms do not provide for satisfactory functional recovery, especially after severe injuries. Peripheral nerve problems are common and encompass a large spectrum of traumatic injuries, diseases, tumors, and iatrogenic lesions. The incidence of traumatic injuries is estimated as $>500,000$ new patients annually in the world. PNI results in loss of neural control in denervated segments of the body, and severe disabilities for the patients. Nerve regeneration usually does not allow for adequate target reinnervation and functional restitution. Neuronal response and axonal regeneration imply a complex interaction of cell types and changes in the expression of many molecules. Many experimental models have been used to gain knowledge on nerve regeneration and to develop strategies to promote recovery.

The failure of axons to regenerate following PNI results from decreased intrinsic properties of the neurons, ${ }^{[3]}$ the absence of neurotrophic factors, or the presence of inhibitory factors in the environment. After PNI, axons and myelin sheaths distal to the lesion are degraded. The degenerative products are eliminated by the cooperative action of denervated Schwann cells and infiltrating macrophages. Wallerian degeneration serves to create a microenvironment favoring axonal regrowth. Schwann cells within the endoneurial tubes of the distal nerve dedifferentiate toward a non-myelinating proliferative phenotype that overexpresses growth factors, cell adhesion molecules, and extracellular matrices. The axotomized neurons shift from a "transmitter" state to a "regenerative" state, so their axons generate growth cones that progress from the proximal stump into the distal nerve. Axonal regeneration requires an adequate substrate of trophic factors, provided by reactive Schwann cells, macrophages, and the extracellular matrix within the degenerated nerve. The regenerative process, however, usually neither reconstitutes a normal nerve structure nor allows for normal distal reconnection after severe lesions. Neuronal response and axonal regeneration require a complex interaction of several cell types and changes in the expression of many molecules with variable spatial and temporal patterns. Therefore, a wide variety of methods are used in experimental studies, depending on the specific goals of each study. ${ }^{[4]}$

\section{Sources of NSCs for clinical application}

Cell replacement therapy could aid in alleviating symptoms or even reversing the progression of neurodegenerative diseases. Over the last decade, convincing evidence has emerged of the capability of various stem cell populations to induce regeneration in animal models of PD, HD, and AD, along with multiple sclerosis and cerebral ischemia. ${ }^{\left[{ }^{[3}\right.}$ Neural stem cells (NSCs) hold tremendous potential for neurodegenerative diseases. This approach is not limited to the use of NSCs for transplantation, but includes the stimulation of endogenous stem cells and the multiple bioactive molecules that they express during reciprocal interactions with the diseased central and peripheral nerve systems. Several critical problems for NSC clinical application remain to be resolved: (1) the source of NSCs should be personalized; (2) the isolation and differentiation protocols of human NSCs should be standardized; (3) the clinical efficacy of NSC transplants must be evaluated in more adequate animal models; and (4) the mechanism of intrinsic brain repair needs to be better characterized. In addition, the ideal imaging technique for tracking NSCs would be safe and yield high temporal and spatial resolution, and good sensitivity and specificity. 
The ideal sources of NSCs for clinical application should better be personalized, tolerant to immune rejection, resistant to tumorigenesis, easy to obtain and amplify, and effective in cellular or molecular replacement. The potential cell sources for central nervous system transplantation include fetal or adult NSCs, ${ }^{[6,7]}$ embryonic stem cells (ESCs), and mesenchymal stem cells (MSCs). NSCs can be expanded over a long period of time, and as they are already neuralized (e.g., committed to a central nervous system cell fate), there is no need for recapitulating early developmental signals that lead to neuroectodermal commitment. However, the transplantation of fetal NSCs into the adult brain is accompanied by numerous ethical, scientific, and legislative hurdles. ${ }^{[8]}$ In addition, the prolonged culturing of NSCs leads to an ever-increasing glial differentiation pattern at the expense of neuronal differentiation, which significantly reduces the therapeutic potential of fetal NSCs ${ }^{[9]}$ Human ESCs can be manipulated to generate defined neuronal and glial lineages, thereby offering a major opportunity to study neurodevelopment and model neurological disease in vitro, as well as potentially having direct therapeutic applications in the field of regenerative neurology. However, certain challenges remain to be resolved before the promise of ESCs for neurological diseases can be fully realized, including the need to optimize survival, fate, and function of neural derivatives upon both neural conversion and long-term differentiation in vitro and in vivo. Current protocols for the derivation of NSCs from ESCs require defined conditions; however, these conditions lead to significant cell death, in which the production of reactive oxygen species has a central role. Thus, neuralization protocols often contain antioxidants (which may increase the propensity to accumulate genetic mutations), involve co-culture with stromal feeder layers, or use B27 and/or N2 supplements. It is becoming increasingly clear that the more traditional stem cell systems using oxygen levels approximating room air (20\%) are far from optimal, particularly with regard to neural specification and differentiation. Thus, recent protocol has been designed to generate NSCs and their regionally specified derivatives from ESCs using a physiological oxygen level of 3\% (normoxia). ${ }^{[10]}$

\section{Isolation and characterization of NSCs}

Flow cytometry and fluorescence-activated cell sorting (FACS) have been used successfully to resolve the complexity of lineage progress in the hematopoietic and other systems. Recently, FACS has been applied extensively in NSC biology, such as isolation of different precursor and progenitor populations from the central nervous system and peripheral nervous system. NSCs were isolated from brain tissues by cell-surface markers (such as CD133) or green fluorescent protein (GFP) expression driven by NSC- specific promoters. These promoters include Sox1, Sox2, Nestin, and fibroblast growth factor 1 (FGF1). ${ }^{[11,12]}$ NSCs thus isolated were cultured in the presence of growth factors and examined to determine whether they could expand to form neurospheres. The capacity to form neurospheres was defined as self-renewal. The potential for neural differentiation of these isolated cells upon withdrawal of growth factors or administration of inducing factors was used to determine multipotency.

The human FGF1 gene was first cloned by us. ${ }^{[13]}$ FGF1 is expressed in ventral cochlear neurons, olfactory bulbs, and hippocampal neurons, but not in glial cells. ${ }^{[14]}$ The brainspecific FGF-1B promoter is active only in the brain. ${ }^{[15,16]}$ Interestingly, it has been shown that FGF-1B mRNA is upregulated for the maintenance of NSCs in hippocampus dentate gyrus in response to activity-induced neurogenesis. ${ }^{[17]}$ Furthermore, FGF-1B promoter $(-540$ to +31$)$-driven GFP reporter (F1B-GFP) could be used to isolate NSCs with self-renewal and multipotent capacities from human glioblastoma tissues ${ }^{[11]}$ and developing neonatal or adult mouse brains [Figure 1]. ${ }^{[11,12]}$ We have developed a series of patented technology in which NSCs could be isolated as GFP-positive cells when adult mouse brain cells are transfected with F1B-GFP plasmid (USA patent No. 6,984,518, 7,045,678, and $7,745,214)$. This F1B-GFP plasmid comprises the GFP coding sequences driven by the human $F G F 1$ promoter. ${ }^{[1]}$ Notably, F1B-GFP-selected NSCs from mouse brains were able to repair the damaged sciatic nerve of paraplegic rats. Micropatterned nerve conduits, together with NSCs and FGF1, could repair PNI in animals. ${ }^{[18,19]}$ Recently, we further developed a novel material named ultra-nanocrystalline diamond (UNCD) and demonstrated that UNCD can enhance the differentiation of NSCs [Figures 2 and 3] and could be used as the standardized differentiation method of NSCs for clinical application in PNIs. ${ }^{[20,21]}$

\section{Current therapeutic applications of NSCs in neurodegeneration and PNIs}

It is widely anticipated that transplantation of stem/ progenitor cells will provide effective therapies for many neurological diseases and injuries. ${ }^{[22]}$ Numerous encouraging animal studies have shown that stem or progenitor cell treatments can rescue some degree of neurological function after injury. We further summarize the preclinical studies evaluating neural stem cell therapy for peripheral nerve repair in Table 1. Only a few studies have demonstrated direct evidence of cell replacement in injury or disease models, which clearly explain the benefits observed after cell therapy. Many positive outcomes after cell therapy appear to be attributed to the rescue of pre-existing tissue rather than repair or cell replacement per se. The paracrine action of growth factors, cytokines, and hormones that are 


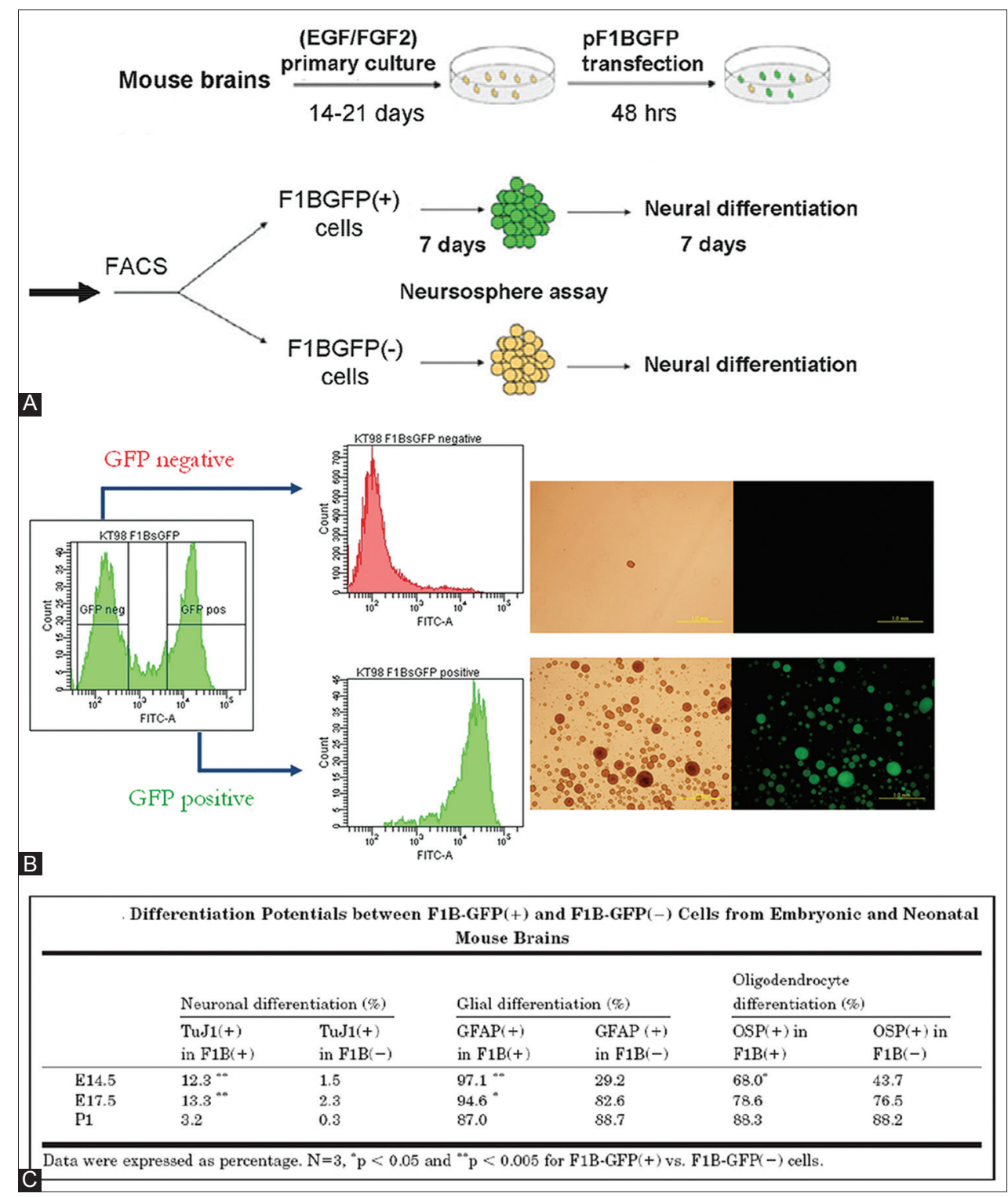

Figure 1: (A) F1B-GFP permits the isolation of $\mathrm{F} 1 \mathrm{~B}(+)$ cells from adult mouse brains. Schematic illustration of the process for the isolation of F1B-GFP(+) neural stem cells from adult mouse brains (NSA, neurosphere assay). (B) KT98/F1B-GFP(+) mouse brain cells showed strong fluorescence intensity. GFP-positive cells formed 2500 neurospheres out of $8 \times 104$ cells plated (3\%), while GFP-negative cells formed 26 spheres $(0.03 \%)$. A 100 -fold enhancement for the neurosphere forming efficiency was demonstrated for the F1B-GFP(+) cells. (C) Differentiation potentials between F1B-GFP(+) mouse brain cells

secreted or released by transplanted cells has been shown to provide most of the benefits after stem/progenitor cell administration. This can be seen as a problem, since for many years we missed paracrine activity as a principal mechanism in cell therapy and the paracrine mechanism may be complicated. Alternatively, the situation can be viewed as an exciting opportunity to the new cell replacement therapy. Notably, a research group in Taipei Veteran General Hospital developed a novel spinal cord injury repair strategy. Using peripheral nerve grafts and FGF1 improves hind limb locomotor function in spinal cord-transected rats. ${ }^{[23-25]}$ Repaired spinal cords induced the expression of the M2 macrophage marker arginase I 6-14 days after repair and recruited large numbers of M2 macrophages to the graft area 10 days after repair. ${ }^{[26]}$ The group further demonstrated that FGF1 induces IL-4 expression and that nerve grafts induce NGF and BDNF expression in transected spinal cords. A full repair strategy utilizes the beneficial effects of both FGF1 and nerve grafts simultaneously. ${ }^{[26]}$

\section{Personalized regenerative medicine: Patient- specific NSCs and functional neurons}

The promising cell sources for personalized regenerative medicine is from patients' skin fibroblasts or even 


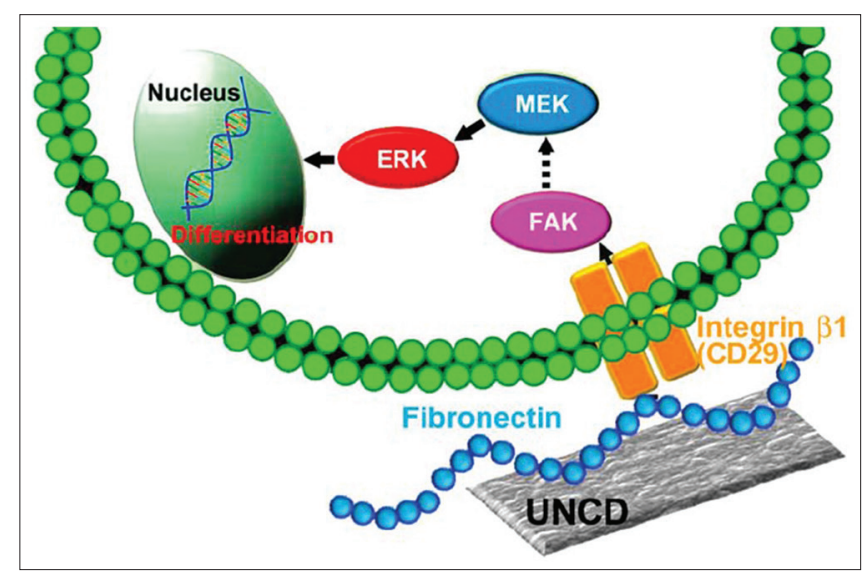

Figure 2: Schematic drawing summarizes the role of H-UNCD films in mediating differentiation from NSCs. Absorbed fibronectin on H-UNCD surface activates integrin, Fak, and Erk1/2 pathways and, in turn, leads to an ultimate specification of neuronal differentiation from NSCs peripheral blood cells through transcription factor-mediated reprogramming, such as induced pluripotent stem (iPS) cells. ${ }^{[27]}$ Development of iPS cells from patients with ALS further attests the possibility of future cell-based therapy. ${ }^{[28]}$ However, recent studies using iPS cells have shown sizeable genetic and epigenetic abnormalities in iPS cells. ${ }^{[29]}$ Recently, Vierbuchen, et al., found that a combination of just three factors (ASCL1, BRN2, and either MYT1L or ZIC1) was sufficient to convert fibroblasts into neurons (iN). ${ }^{[30]}$ These iN cells expressed a variety of neuronal markers and were capable of firing action potentials. Furthermore, when cultured with mouse neural cells, the iN cells received both excitatory and inhibitory synaptic connections from the mouse neurons, and were able to form functional synapses with each other. ASCL1 alone was able to produce cells with immature neuronal features, but co-infection with

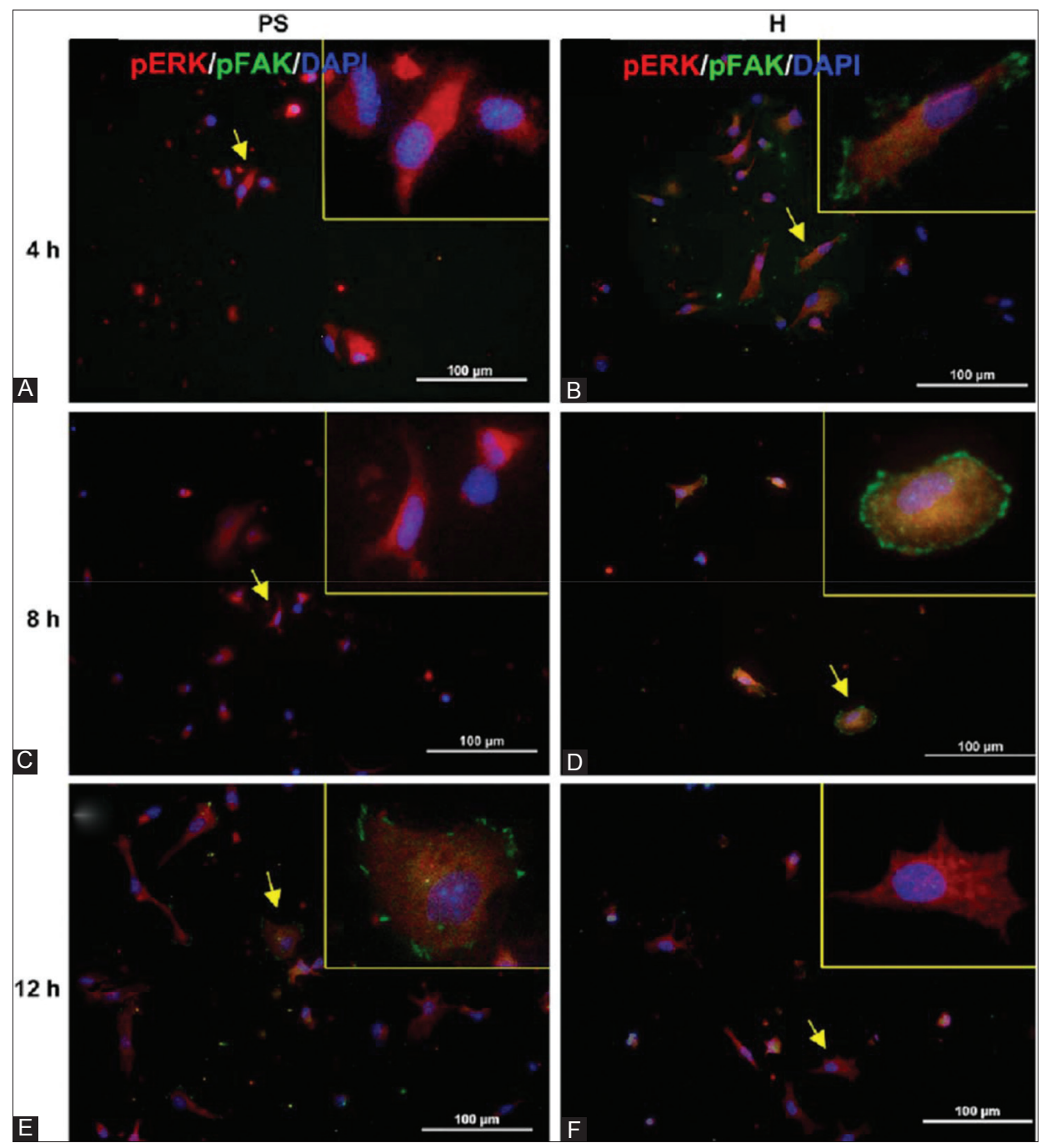

Figure 3: Erk1/2 and F1k signaling pathways were activated during neuronal differentiation that was induced by $H$-UNCD films. The left panels show the phosphorylated Erk1/2 and phosphorylated Fak (A, C, E) cultured on polystyrene, while the right panels (B, D, F) show the NSCs cultured on H-UNCD films (H). Immunofluorescence triple labeling shows staining patterns of pErk (red), pFak (green), and DAPI (blue) on NSCs at three different time points: $4 \mathrm{~h}(\mathrm{a}, \mathrm{b}), 8 \mathrm{~h}(\mathrm{C}, \mathrm{D})$, and $12 \mathrm{~h}(\mathrm{E}, \mathrm{F})$ 
$B R N 2$ and $Z I C 1$ was required to produce cells with more mature neuronal features. In the past 6 months, six different laboratories independently demonstrated that adult human fibroblasts from a skin biopsy could be efficiently converted into functional neurons. ${ }^{[31]}$

We have successfully generated iN cells from human dermal fibroblast cells using MIR124, BRN2, and MYT1L [Figure 4]. We are currently testing if more accessible cell types such as peripheral blood could be reprogrammed into iN cells. This is a reasonable hypothesis since reprogramming of blood cells to iPS cells has been successfully demonstrated. ${ }^{[32]}$ Notably, a way of sustaining cell growth and expanding cell numbers is required. Also, FGF1 has been shown to be essential in culturing ESCs and NSCs. FGF1 has been shown to sustain neuron survival and induce neurite outgrowth. It is of great interest to note that Vierbuchen et al., have shown that ASCL1 alone was able to produce cells with immature neuronal features ${ }^{[30]}$ and co-infection with ZIC1 was required to produce cells with more mature neuron al features. ZIC1 and ZIC2 share highly conserved tandem repeats known as $\mathrm{C} 2 \mathrm{H} 2$ zinc finger. Thus, it is exciting that our preliminary data showed that ZIC2 could bind to the 26-bp cis-element of FGF1 gene 1B promoter and activate the dual reporter in yeast one-hybrid screening. Our previous study suggested that endogenous FGF1 is crucial for the self-renewal of adult NSCs. ${ }^{[33]}$ Therefore, we believe that human dermal fibroblast cells and peripheral blood cells could be reprogrammed into iN cells by using ZIC 2 in combination with other transcription factors and miRNAs.
If iN conversion is made possible by ZIC2, it might help expand the cell number of iN cells by making use of the advantage of FGF1. It is likely that these iN cells could be applied in cell therapy, drug screening, and mechanism studies. Furthermore, F1B-GFP reporter gene will be used as an approach to screen small molecules that would allow F1B-GFP expression in iNs derived from patients with neurodegenerative diseases and nerve injuries. These small molecules might be able to enhance the neuronal circuitry that is defective in these neurological disorders. Successful generation of patient-specific neurons will provide in vitro disease models to study signaling pathways. These
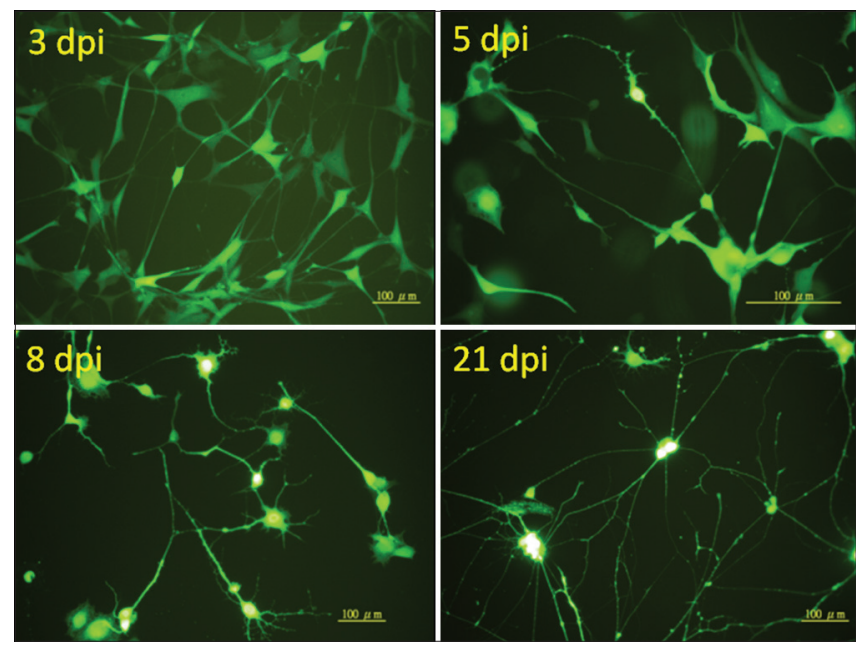

Figure 4: Generation of iN cells from human dermal fibroblast cells using MIR124, BRN2, and MYT1L (dpi, days post infection)

Table 1: Preclinical studies evaluating stem cell therapy for peripheral nerve repair

\begin{tabular}{|c|c|c|c|}
\hline Study & Injury type/animal model & Cellular type/factor & Major findings \\
\hline Dezawa et al. (2001) & $\begin{array}{l}\text { Sciatic nerve injury in rats } \\
(1.5 \mathrm{~cm} \text { gap })\end{array}$ & $\begin{array}{l}\text { Bone marrow MSCs differentiated into } \\
\text { Schwann-like cells suspended in Matrigel } \\
\text { injected into hollow fibers }\end{array}$ & $\begin{array}{l}\text { Successful nerve regeneration and } \\
\text { myelination }\end{array}$ \\
\hline McKenzie et al. (2006) & $\begin{array}{l}\text { Sciatic nerve crush injury } \\
\text { in myelin-deficient mice }\end{array}$ & $\begin{array}{l}\text { Skin-derived precursors differentiated } \\
\text { into Schwann cells }\end{array}$ & Remyelination and functional recovery \\
\hline Marchesi et al. (2007) & $\begin{array}{l}\text { Sciatic nerve injury in rats } \\
(1.6 \mathrm{~cm} \text { gap })\end{array}$ & Guides filled with skin-derived stem cells & Functional recovery and myelination \\
\hline Cui et al. (2008) & $\begin{array}{l}\text { Sciatic nerve injury in rats } \\
(1.0 \mathrm{~cm} \text { gap })\end{array}$ & $\begin{array}{l}\text { Mouse ES cell-derived neural progenitor } \\
\text { cells }\end{array}$ & Remyelination and functional recovery \\
\hline Lin et al. (2008) & Sciatic nerve injury in rats & Nerve conduit seeded with F1BGFP(+) & Axon regeneration \\
\hline Hsu et al. (2009a) & $(1.0 \mathrm{~cm}$ gap $)$ & mouse neural stem cells & \\
\hline Chen et al. (2010) & $\begin{array}{l}\text { Acutely distracted sciatic } \\
\text { nerves in rabbits }\end{array}$ & Neural stem cells & Functional recovery and nerve regeneration \\
\hline Gu et al. (2010) & $\begin{array}{l}\text { Sciatic nerve injury in rats } \\
(1.0 \mathrm{~cm} \text { gap })\end{array}$ & Rat fetal neural stem cells & $\begin{array}{l}\text { Transplanted neural stem cells differentiated } \\
\text { into neurons in peripheral nerves that } \\
\text { synthesize and secreted synaptophysin }\end{array}$ \\
\hline Cheng et al. (2010) & $\begin{array}{l}\text { Sciatic nerve crush injury } \\
\text { in rats }\end{array}$ & $\begin{array}{l}\text { Human amniotic fluid-derived } \\
\text { mesenchymal stem cells and }\end{array}$ & Functional recovery and nerve regeneration \\
\hline Di Summa et al. (2010) & $\begin{array}{l}\text { Sciatic nerve injury in rats } \\
(1.0 \mathrm{~cm} \text { gap })\end{array}$ & $\begin{array}{l}\text { Nerve fibrin conduits seeded with } \\
\text { adipose derived stem cells }\end{array}$ & Enhanced peripheral nerve repair \\
\hline Reid et al. (2011) & $\begin{array}{l}\text { Sciatic nerve injury in rats } \\
(1.0 \mathrm{~cm} \text { gap })\end{array}$ & Adipose derived stem cells & Dorsal root ganglia protection from apoptosis \\
\hline
\end{tabular}


induced neurons will provide the means to screen for drugs that might ameliorate neurological pathologies. Thus, the identification of small molecules that sustain neuronal cells will have a significant impact in the multi-billion dollar market of neural diseases, including nerve injury and neurodegenerative disorders.

In addition, combination of FGF1 and the iN cells is also promising for future therapeutic application. As a proof of principle, Cheng, et al., and Lee, et al., showed that the combination of FGF1 treatment and peripheral nerve grafts could restore hind limb function in adult paraplegic rats. ${ }^{[23,24]}$ More recently, it was shown that similar treatments benefited patients with common peroneal nerve lesions and spinal cord injury as well. ${ }^{[34,35]}$ Thus, in addition to cell-based therapies, direct clinical application of FGF1 will likely benefit the patients. Given the significance of FGF1 in the treatment of spinal cord injury and PNI, future efforts generating FGF1expressing NSCs or iNs will have promising potential in regenerative medicine and in the treatment of central and peripheral nerve system diseases.

\section{REFERENCES}

1. Zlokovic BV. Neurovascular pathways to neurodegeneration in Alzheimer's disease and other disorders. Nat Rev Neurosci 2011;12:723-38

2. Kish SJ, Shannak K, Hornykiewicz O. Uneven pattern of dopamine loss in the striatum of patients with idiopathic Parkinson's disease. Pathophysiologic and clinical implications. N Engl J Med 1988;318:876-80.

3. Kadoya K, Tsukada S, Lu P, Coppola G, Geschwind D, Filbin MT, et al. Combined intrinsic and extrinsic neuronal mechanisms facilitate bridging axonal regeneration one year after spinal cord injury. Neuron 2009;64:165-72.

4. Navarro X. Chapter 27: Neural plasticity after nerve injury and regeneration. Int Rev Neurobiol 2009;87:483-505.

5. Gogel S, Gubernator M, Minger SL. Progress and prospects: Stem cells and neurological diseases. Gene Ther 2011;18:1-6.

6. Tamaki S, Eckert K, He D, Sutton R, Doshe M, Jain G, et al. Engraftment of sorted/expanded human central nervous system stem cells from fetal brain. J Neurosci Res 2002;69:976-86.

7. Uchida N, Buck DW, He D, Reitsma MJ, Masek M, Phan TV, et al. Direct isolation of human central nervous system stem cells. Proc Natl Acad Sci USA 2000;97:14720-5.

8. Mathews DJ, Sugarman J, Bok H, Blass DM, Coyle JT, Duggan P, et al. Cell-based interventions for neurologic conditions: Ethical challenges for early human trials. Neurology 2008;71:288-93.

9. Anderson L, Burnstein RM, He X, Luce R, Furlong R, Foltynie T, et al. Gene expression changes in long term expanded human neural progenitor cells passaged by chopping lead to loss of neurogenic potential in vivo. Exp Neurol 2007;204:512-24.

10. Stacpoole SR, Bilican B, Webber DJ, Luzhynskaya A, He XL, Compston A, et al. Efficient derivation of NPCs, spinal motor neurons and midbrain dopaminergic neurons from $\mathrm{hESCs}$ at $3 \%$ oxygen. Nat Protoc 2011;6:1229-40.
11. Hsu YC, Lee DC, Chen SL, Liao WC, Lin JW, Chiu WT, et al. Brainspecific $1 \mathrm{~B}$ promoter of FGF1 gene facilitates the isolation of neural stem/progenitor cells with self-renewal and multipotent capacities. Dev Dyn 2009;238:302-14.

12. Lee DC, Hsu YC, Chung YF, Hsiao CY, Chen SL, Chen MS, et al. Isolation of neural stem/progenitor cells by using EGF/FGF1 and FGF1B promoter-driven green fluorescence from embryonic and adult mouse brains. Mol Cell Neurosci 2009;41:348-63.

13. Wang WP, Lehtoma K, Varban ML, Krishnan I, Chiu IM. Cloning of the gene coding for human class 1 heparin-binding growth factor and its expression in fetal tissues. Mol Cell Biol 1989;9:2387-95.

14. Alam KY, Frostholm A, Hackshaw KV, Evans JE, Rotter A, Chiu IM. Characterization of the 1B promoter of fibroblast growth factor 1 and its expression in the adult and developing mouse brain. J Biol Chem 1996;271:30263-71.

15. Chiu IM, Touhalisky K, Liu Y, Yates A, Frostholm A. Tumorigenesis in transgenic mice in which the SV40 T antigen is driven by the brain-specific FGF1 promoter. Oncogene 2000;19:6229-39.

16. Chiu IM, Touhalisky K, Baran C. Multiple controlling mechanisms of FGF1 gene expression through multiple tissue-specific promoters. Prog Nucleic Acid Res Mol Biol 2001;70:155-74.

17. Ma DK, Jang MH, Guo JU, Kitabatake Y, Chang ML, PowAnpongkul N, et al. Neuronal activity-induced Gadd45b promotes epigenetic DNA demethylation and adult neurogenesis. Science 2009;323:1074-7.

18. Lin YL, Jen JC, Hsu SH, Chiu IM. Sciatic nerve repair by microgrooved nerve conduits made of chitosan-gold nanocomposites. Surg Neurol 2008;70:S1:9-18.

19. Hsu SH, Su CH, Chiu IM. A novel approach to align adult neural stem cells on micropatterned conduits for peripheral nerve regeneration: A feasibility study. Artif Organs 2009;33:26-35.

20. Chen YC, Lee DC, Hsiao CY, Chung YF, Chen HC, Thomas JP, et al. The effect of ultra-nanocrystalline diamond films on the proliferation and differentiation of neural stem cells. Biomaterials 2009;30:3428-35.

21. Chen YC, Lee DC, Tsai TY, Hsiao CY, Liu JW, Kao CY, et al. Induction and regulation of differentiation in neural stem cells on ultra-nanocrystalline diamond films. Biomaterials 2010;31:5575-87.

22. Trounson A, Thakar RG, Lomax G, Gibbons D. Clinical trials for stem cell therapies. BMC Med 2011;9:52.

23. Cheng H, Cao Y, Olson L. Spinal cord repair in adult paraplegic rats: Partial restoration of hind limb function. Science 1996;273:510-3.

24. Lee YS, Hsiao I, Lin VW. Peripheral nerve grafts and aFGF restore partial hindlimb function in adult paraplegic rats. J Neurotrauma 2002;19:1203-16.

25. Tsai MC, Shen LF, Kuo HS, Cheng H, Chak KF. Involvement of acidic fibroblast growth factor in spinal cord injury repair processes revealed by a proteomics approach. Mol Cell Proteomics 2008;7:1668-87.

26. Kuo HS, Tsai MJ, Huang MC, Huang WC, Lee MJ, Kuo WC, et al. The combination of peripheral nerve grafts and acidic fibroblast growth factor enhances arginase I and polyamine spermine expression in transected rat spinal cords. Biochem Biophys Res Commun 2007;357:1-7.

27. Takahashi K, Tanabe K, Ohnuki M, Narita M, Ichisaka T, Tomoda K, et al. Induction of pluripotent stem cells from adult human fibroblasts by defined factors. Cell 2007;131:861-72. 
28. Dimos JT, Rodolfa KT, Niakan KK, Weisenthal LM, Mitsumoto H, Chung W, et al. Induced pluripotent stem cells generated from patients with ALS can be differentiated into motor neurons. Science 2008;321:1218-21.

29. Pera MF. Stem cells: The dark side of induced pluripotency. Nature 2011;471:46-7.

30. Vierbuchen T, Ostermeier A, Pang ZP, Kokubu Y, Sudhof TC, Wernig M. Direct conversion of fibroblasts to functional neurons by defined factors. Nature 2010;463:1035-41.

31. Yang N, Ng YH, Pang ZP, Sudhof TC, Wernig M. Induced neuronal cells: How to make and define a neuron. Cell Stem Cell 2011;9:517-25
32. Yamanaka S. Patient-specific pluripotent stem cells become even more accessible. Cell Stem Cell 2010;7:1-2.

33. Hsu YC, Kao CY, Chung YF, Chen MS, Chiu IM. Regulation of FGF1 Gene Promoter through Transcription factor RFX1. J Biol Chem 2010;235:13885-95.

34. Tsai PY, Cheng H, Huang WC, Huang MC, Chiu FY, Chang YC, et al. Outcomes of common peroneal nerve lesions after surgical repair with acidic fibroblast growth factor. J Trauma 2009;66:1379-84.

35. Wu JC, Huang WC, Chen YC, Tu TH, Tsai YA, Huang SF, et al. Acidic fibroblast growth factor for repair of human spinal cord injury: A clinical trial. J Neurosurg Spine 2011;3:216-27. 